\title{
Avaliação Florística do Estrato Regenerante de Reflorestamentos em Área Reabilitada na Mata Atlântica
}

\author{
Joana Farias dos Santos ${ }^{1}$, Ricardo Valcarcel ${ }^{2}$ \\ ${ }^{1}$ Programa de Ciências Ambientais e Florestais, Universidade Federal Rural do Rio de Janeiro - UFRRJ \\ ${ }^{2}$ Departamento de Ciências Ambientais, Instituto de Florestas, Universidade Federal Rural do Rio de Janeiro - UFRRJ
}

\section{RESUMO}

A regeneração espontânea sob reflorestamentos indica a eficácia da sucessão ecológica e a sustentabilidade da reabilitação. A área de empréstimo estudada foi reabilitada há 14 anos, a partir de cinco reflorestamentos com espécies diferentes pela proporcionalidade e pelas propriedades funcionais. Avaliou-se a composição florística e a riqueza do estrato regenerante ( $\mathrm{h} \geq 15 \mathrm{~cm} ;$ CAP $\leq 10 \mathrm{~cm}$ ), utilizando-se o método de pontos, no inverno (2007) e no verão (2008). Encontraram-se 3.554 indivíduos, 38 famílias, 84 gêneros e 147 espécies. Dentre as famílias botânicas presentes, Asteraceae, Poaceae e Fabaceae-Mimosoideae foram as principais. O Tratamento T5 com predomínio da Mimosa caesalpiniaefolia Benth apresentou maior riqueza no verão (29) e inverno (41), sendo o melhor de todos os tratamentos. O menor valor no verão foi para T3 (28), com predomínio de Acacia mangium Willd, e no inverno o menor valor foi para T4 (19), com predomínio de Acacia auriculiformis Sandw. Houve evolução dos ecossistemas reabilitados na busca da sustentabilidade ambiental, quando comparados com a área testemunha (T0).

Palavras-chave: área degradada, regeneração, sucessão vegetal.

\section{Floristic Evaluation of the Regenerating Stratum of Reforestations in a Rehabilitated Atlantic Forest Area}

\begin{abstract}
Spontaneous regeneration in reforestation areas can indicate effectiveness of ecological succession and rehabilitation sustainability. The studied recovery area had been rehabilitated 14 years before, with five reforestations with different species with proportional and functional properties. The floristic composition and richness of the regenerating stratus was evaluated $(\mathrm{h} \geq 15 \mathrm{~cm} ; \mathrm{DBH} \leq 10 \mathrm{~cm})$ using point methodology in the winter $(2007)$ and the summer (2008). There were found 3,554 individuals, 38 families, 84 genus and 147 species. The main families were: Asteraceae, Poaceae and Fabaceae-Mimosoideae. Treatment T5 with predominance of Mimosa caesalpiniaefolia Benth presented the greatest species richness in summer (29) and in winter (41). Treatment T3 presented the lowest value (28), with predominance of Acacia mangium Willd; and, in winter, T4 (19), with predominance of Acacia auriculiformis Sandw. There was an improvement of the rehabilitated ecosystem in sustainability path when compared to the witness area (T0).
\end{abstract}

Keywords: degraded areas, regeneration, vegetal succession. 


\section{INTRODUÇÃO}

O crescimento populacional implica no aumento da demanda dos recursos naturais, além de afetar os serviços ambientais dos ecossistemas, principalmente se houver processos de degradação (Valcarcel et al., 2007).

Os fatores econômicos que podem gerar degradação ambiental são variados, sendo a mineração um dos mais reconhecidos, pois alia alta intensidade de degradação e pequenas áreas de atuação, formando áreas de empréstimo (Pinheiro, 2004). Nessas áreas, se promovem a supressão da vegetação e a remoção de solos e subsolos, levando à alteração de todo o ecossistema (Pinheiro, 2004). Esse processo leva à formação de áreas degradadas, onde a probabilidade de recuperação espontânea em período de uma geração humana é baixa, demandando assim intervenções externas (Valcarcel \& Silva, 2000)

Na Ilha da Madeira, Itaguaí, RJ, em meados do século XVI, houve supressão da floresta nativa (Valcarcel \& Silva, 2000), a qual foi substituída por pastagens, seguidas pela atividade de mineração do substrato para construção do retroporto de Itaguaí e obtenção de brita para a construção civil. Após 14 anos do encerramento dessa exploração do substrato, a área não apresentou crescimento espontâneo da vegetação e nem indício de estabilização de processos erosivos, sendo necessária a realização de medidas físicas, físico-biológicas e biológicas para iniciar os processos de reabilitação (Universidade..., 1993).

Reconstruir ecossistemas com funções similares às originais e formas compatíveis com as do contexto regional constitui as atividades perseguidas pela reabilitação de áreas degradadas (Valcarcel \& Silva, 2000). Os usos das espécies vegetais como elemento construtor desses ecossistemas, associando suas habilidades, constituem um dos principais desafios tecnológicos da reabilitação sustentável (Bechara et al., 2007).

Os reflorestamentos com espécies rústicas podem garantir a regeneração espontânea e estas, o desenvolvimento e a dinâmica das florestas com seus processos sucessionais (Macedo, 1993).

Como o reflorestamento da área de estudo foi efetuado envolvendo cinco combinações de espécies
- medidas biológicas (tratamentos), que diferem entre si pela proporcionalidade entre espécies - , a eficácia dos processos de sucessão ecológica vai depender da combinação de funções dessas espécies, melhorando a oferta de atributos ambientais aos ecossistemas em construção.

Este estudo teve como objetivo avaliar a composição florística e a riqueza de espécies nos estratos regenerantes de cinco reflorestamentos utilizados como medidas biológicas para reabilitação de área de empréstimo degradada.

\section{MATERIAL E MÉTODOS}

A área localiza-se na região da Costa Verde, Distrito da Ilha da Madeira (latitude $23^{\circ} 55^{\prime} 07^{\prime \prime} \mathrm{S}$ e longitude $43^{\circ} 49^{\prime} 73^{\prime \prime} \mathrm{O}$ ), Município de Itaguaí, RJ, aos fundos da Baía de Sepetiba, sobre morrote de encostas com feições de relevo regulares, declividade média de 30\%, cujo cume alcança $220 \mathrm{~m}$. Pertence ao domínio ecológico do bioma Mata Atlântica no ecossistema Floresta Ombrófila Densa, clima "Aw" tropical, quente e úmido, solos Argissolos nas vertentes e Gleissolos nas várzeas (Universidade..., 1993). O período de maior pluviosidade concentrase entre dezembro e janeiro, enquanto o período seco estende-se de maio a setembro, sendo a pluviosidade média de 1500 mm/ano (Rio de Janeiro..., 1996).

A supressão total da vegetação nativa, da fauna e dos solos foi feita em uma área de 10,81 ha, sendo retirados $1,4 \times 10^{6} \mathrm{~m}^{3}$ de substrato de 1977 a 1979 , com profundidade média de $13 \mathrm{~m}$ de decape. De 1980 a 1993, a área foi abandonada às intempéries da natureza, sofrendo intensos processos erosivos.

Em 1994, foram implantadas cinco medidas biológicas (reflorestamentos), cujo plantio foi efetuado em curva de nível, distribuição aleatória das espécies, densidade de mudas similares, feito em covas $(0,40 \times 0,40 \times 0,40 \mathrm{~m})$ com $2 \mathrm{~L}$ de esterco/ cova, espaçamento médio de $2 \mathrm{~m}$, equivalente a 2.020 plantas/ha (Universidade..., 1993). Tais medidas biológicas combinaram espécies com funções ecológicas distintas (tratamentos) e diferiram na proporção de espécies exóticas e nativas (Universidade..., 1993) (Tabela 1).

O presente estudo de caso foi realizado sobre área de empréstimo similar às existentes na 
Tabela 1. Composição percentual das espécies plantadas em 1994 como Medidas Biológicas (tratamentos) na área de empréstimo da Ilha da Madeira, Itaguaí. RJ.

Table 1. Percentage composition of planted species on 1994 as Biological Measures (treatments) on a reclaimed area at Ilha da Madeira, Itaguaí, RJ.

\begin{tabular}{|c|c|c|c|c|c|c|c|}
\hline Espécies utilizadas & Nome popular & T0 & T1 & T2 & T3 & T4 & T5 \\
\hline \multicolumn{8}{|l|}{ Fabaceae } \\
\hline Acacia auriculiformis Sandw ${ }^{1}$ & acácia & 0 & 100 & 25 & 0 & 22 & 0 \\
\hline Acacia mangium Willd ${ }^{1}$ & acácia & 0 & 0 & 0 & 30 & 17 & 0 \\
\hline Albizia lebbeck (L) Benth. ${ }^{1}$ & albizia & 0 & 0 & 7 & 20 & 0 & 0 \\
\hline Caesalpinia ferrea Mart. $^{3}$ & pau-ferro & 0 & 0 & 13 & 0 & 0 & 0 \\
\hline Clitoria fairchildiana Howard ${ }^{2}$ & sombreiro & 0 & 0 & 0 & 20 & 20 & 17 \\
\hline Inga laurina (Sw.) Willd. ${ }^{3}$ & ingá & 0 & 0 & 0 & 0 & 20 & 15 \\
\hline Leucaena leucocephala (Lam.) ${ }^{1}$ & leucaena & 0 & 0 & 20 & 0 & 0 & 15 \\
\hline Mimosa bimucronata (DC.) ${ }^{2}$ & maricá & 0 & 0 & 0 & 0 & 0 & 13 \\
\hline Mimosa caesalpiniaefolia Benth $^{2}$ & sabiá & 0 & 0 & 10 & 30 & 0 & 21 \\
\hline Piptadenia gonoacantha (Mart.) J.F. Macbr. ${ }^{2}$ & pau-jacaré & 0 & 0 & 15 & 0 & 10 & 5 \\
\hline \multicolumn{8}{|l|}{ Urticaceae } \\
\hline Cecropia pachystachya Trec. $^{2}$ & embaúba & 0 & 0 & 0 & 0 & 0 & 8 \\
\hline \multicolumn{8}{|l|}{ Myrtaceae } \\
\hline Psidium guajava L. ${ }^{2}$ & goiabeira & 0 & 0 & 5 & 0 & 11 & 0 \\
\hline \multicolumn{8}{|l|}{ Anacardiaceae } \\
\hline Schinus terebinthifolius Raddi $^{2}$ & aroeira-vermelha & 0 & 0 & 0 & 0 & 0 & 6 \\
\hline \multicolumn{8}{|l|}{ Bignoniaceae } \\
\hline Tabebuia umbellata (Sonder) Landwith ${ }^{3}$ & ipê-amarelo & 0 & 0 & 5 & 0 & 0 & 0 \\
\hline Total de especies & & 0 & 1 & 8 & 4 & 6 & 8 \\
\hline Total de exóticas (\%) & & 0 & 100 & 52 & 50 & 39 & 15 \\
\hline Total de nativas (\%) & & 0 & 0 & 48 & 50 & 61 & 85 \\
\hline
\end{tabular}

Fonte: Universidade... (1993), modificado. Nota: 1) pioneira exótica; 2) pioneira nativa; 3) secundária nativa.

região, inclusive em suas reduzidas dimensões. Reflorestamentos heterogêneos com mesmas composições de espécies, implantados em sítios ambientalmente similares em termos de ofertas de fatores ecológicos, são condições básicas para se avaliarem os efeitos das interações bióticas nos processos de reabilitação de áreas degradadas, além de serem fatores limitantes para o estabelecimento de repetições.

A área experimental foi estabelecida no interior dos reflorestamentos, em parcelas de $25 \times 50 \mathrm{~m}$, subdividida em quatro subparcelas permanentes de $5 \times 15 \mathrm{~m}\left(75 \mathrm{~m}^{2}\right)$, separadas entre si por $1 \mathrm{~m}$. Foi alocada uma área testemunha, que teve a mesma área e subparcelas dos reflorestamentos, porém sem plantios. Todas as parcelas e a área testemunha apresentam características litólicas e hidrogeoambientais similares.

Foram considerados como estrato regenerante todos os indivíduos das parcelas acima de $15 \mathrm{~cm}$ de altura e CAP $\leq 10 \mathrm{~cm}$. O levantamento foi realizado sazonalmente, no inverno (julho de 2007) e verão (fevereiro de 2008), de modo a avaliar a dinâmica sucessional em períodos com ofertas diferenciadas de umidade, por meio do método fitossociológico de Pontos (Cockaine, 1926, adaptado por Mantovani, 1987).

Em cada parcela e na área testemunha, foram levantados 300 pontos e as espécies encontradas foram classificadas de acordo com o APGII (Angiosperm..., 2003).

Diferenças entre os tratamentos com relação à riqueza de espécies foram analisadas por meio do teste de Kruskal-Wallis (Zar, 1999), utilizando-se o pacote computacional estatístico R ( R Develoment Core Team, 2007).

A diversidade florística foi avaliada pelo cálculo do Índice de Diversidade de Shannon-Weaver ( $\left.\mathrm{H}^{\prime}\right)$, conforme Brower \& Zar (1984). 


\section{RESULTADOS E DISCUSSÃO}

Foram amostrados 3.554 indivíduos, sendo 1.438 no inverno e 2.116 no verão, pertencentes a 38 famílias, 84 gêneros e 147 espécies (Tabela 2 e Anexo 1). Em levantamento realizado em 2000, no verão, no outono, no inverno e na primavera, na mesma área de estudo, foram encontrados 3.562 indivíduos da regeneração, distribuídos em 94 espécies pertencentes a 75 gêneros e 31 famílias (Neves, 2004). Esses dados evidenciam que houve incremento na biodiversidade em nível de indivíduos, espécies, gêneros e famílias, quando comparados com os resultados atuais, havendo maior diversidade de gêneros e espécies no inverno e no verão.

As famílias botânicas mais representativas foram Asteraceae (21 espécies), Poaceae (15 espécies) e Fabaceae-Mimosoideae (10 espécies) (Figura 1).

A área testemunha (T0) apresentou todos os valores abaixo dos encontrados nos tratamentos, no inverno e no verão, para número de famílias, gêneros e espécies acumuladas (Figuras 2 e 3 ) e menor valor para indivíduos encontrados no inverno (Tabela 2). Os tratamentos T2 e T5 apresentaram, no inverno, maior número de famílias (ambos $\mathrm{n}=17$ ) e $\mathrm{T} 4$ apresentou menor número, com nove famílias. Já no verão, o T5 teve o maior número de famílias $(\mathrm{n}=23)$ e o T3, o menor $(\mathrm{n}=15)$ (Figura 2).

Os resultados podem estar refletindo diferenças entre as combinações de espécies do reflorestamento inicial quanto à administração da entrada de água das chuvas, em razão do arranjo diferenciado de suas copas. Estas podem ocasionar ofertas diferenciadas de funções ambientais aos ecossistemas, organismos polinizadores, dispersores e predadores naturais
(Moraes, 2006), além de possíveis efeitos sinergismos entre as espécies, as quais atuam como engenheiras dos ecossistemas, especializando-se em explorar os seus recursos de forma diferenciada e em disponibilizá-los ao meio para que sejam aproveitados (Jones et al., 1997); esse conjunto de características contribui, dessa forma, para que o T5 tenha se diferenciado dos demais tratamentos.
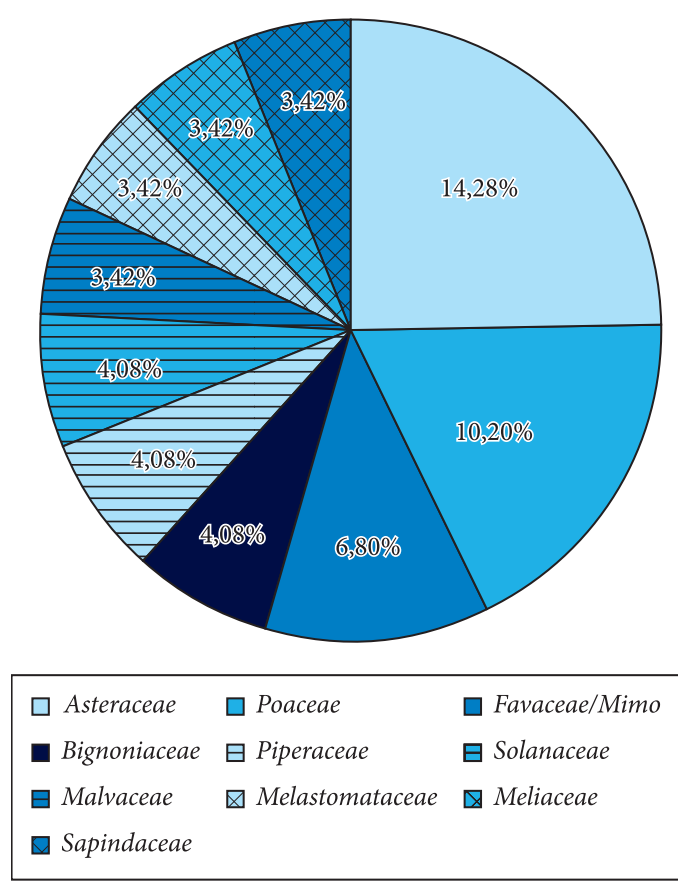

Figura 1. Principais famílias do estrato regenerante nas áreas em reabilitação na Ilha da Madeira, Itaguaí, RJ, reflorestada em 1994

Figure 1. Major families of regenerating stratum on a rehabilitated area at Ilha da Madeira, Itaguaí, RJ, reforested on 1994.

Tabela 2. Número de indivíduos amostrados nos tratamentos T1, T2, T3, T4, T5 e na testemunha T0 em área de empréstimo na Ilha da Madeira, RJ, reflorestada em 1994.

Table 2. Number of individuals surveyed at treatments T1, T2, T3, T4, T5 and withness T0, at a reclamation area at Ilha da Madeira, RJ, reforested on 1994.

Tratamentos

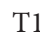

$\mathrm{T} 2$

T3

$\mathrm{T} 4$

$\mathrm{T} 5$

T0

Total
No de Indivíduos amostrados (Inverno)

$\begin{array}{cc}430 & 540 \\ 440 & 482 \\ 237 & 354 \\ 86 & 144 \\ 193 & 330 \\ 52 & 266 \\ 1.438 & 2.116\end{array}$




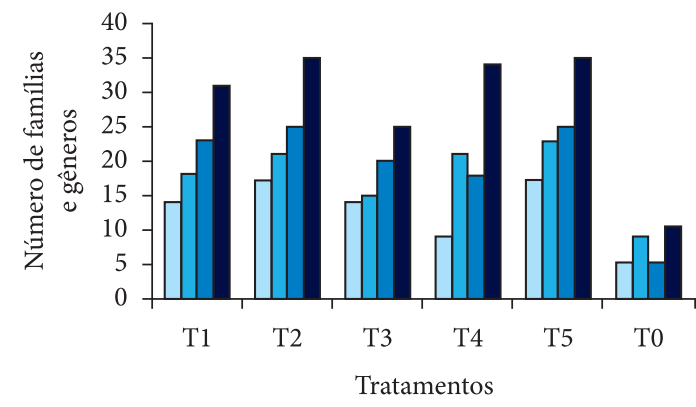

$\square \mathrm{N}^{\circ}$ famílias INV $\square \mathrm{N}^{\circ}$ famílias VER

$\square \mathrm{N}^{\circ}$ gêneros INV $\quad \mathrm{N}^{\circ}$ gêneros VER

Figura 2. Número de famílias e gêneros no inverno (2007) e verão (2008) da regeneração espontânea em área reabilitada na Ilha da Madeira, Itaguaí, RJ, reflorestada em 1994.

Figure 2. Number of families and genus of spontaneous regeneration, on winter (2007) and summer (2008), on a rehabilitated area at Ilha da Madeira, Itaguaí, RJ, reforested on 1994.

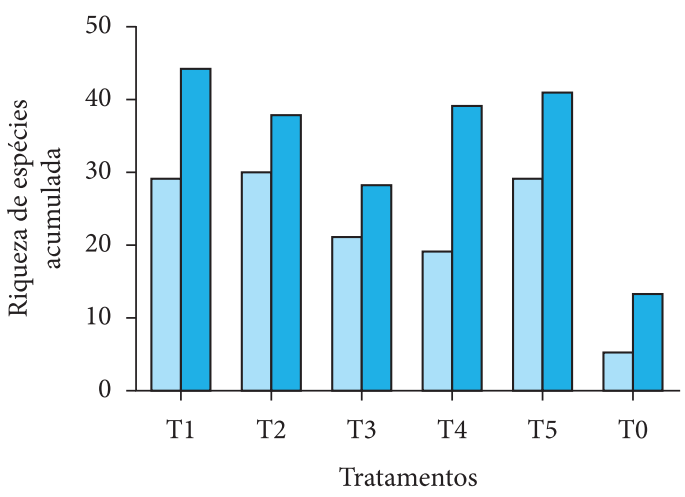

$\square$ Inverno $\square$ Verão

Figura 3. Riqueza de espécies da regeneração espontânea nos tratamentos T1, T2, T3, T4, T5 e na área testemunha (T0) nos períodos de inverno (2007) e verão (2008), em área de empréstimo na Ilha da Madeira, Itaguaí, RJ, reflorestada em 1994.

Figure 3. Species richness from spontaneous regeneration on treatments $\mathrm{T} 1, \mathrm{~T} 2, \mathrm{~T} 3, \mathrm{~T} 4, \mathrm{~T} 5$ and withness area (T0) on winter period (2007) and summer (2008), at a reclamation area at Ilha da Madeira, Itaguaí, RJ, reforested on 1994.

Foram encontradas diferenças estatísticas significativas entre tratamentos e área testemunha $(\mathrm{KW}=824.525 ; \mathrm{P}<0,001)$ do período de inverno para o verão $(\mathrm{KW}=926.201 ; \mathrm{P}<0,001)$ para riqueza acumulada de espécies regenerantes, representando variação da dinâmica sucessional entre os tratamentos e a testemunha, e períodos com maior (verão) e menor umidade (inverno).

A testemunha (T0) apresentou menor riqueza de espécies acumuladas, tanto no inverno (5) como no verão (13) (Figura 3).

Entre os tratamentos, quanto ao acúmulo da riqueza de espécies, no período do inverno, o T4 (19 espécies) teve os menores valores, enquanto que o tratamento T2 (30 espécies) apresentou os melhores resultados, seguido de T5 e T1 (ambos com 29 espécies). No verão, o menor valor foi no T3 (28 espécies) e os maiores, no T1 (44 espécies), seguido do T5 (41) (Figura 3).

Evidencia-se também, de acordo com a Figura 3, que o maior ingresso de novas espécies ocorreu no período de maior unidade (verão) em comparação com o inverno, quando há estresse hídrico. Segundo Valcarcel \& D’Alterio (1988), no período do verão há elevação da temperatura e da umidade, assim como elevação da capacidade de retenção de água, fator limitante para o estabelecimento vegetal em medidas biológicas. Com precipitação regular, aumenta umidade relativa do ar e do solo, favorecendo a germinação e estabelecimento das plântulas (Mattos et al., 1998).

Esses resultados podem ser justificados em função das diferentes combinações de espécies implantadas nos reflorestamentos, que podem ter permitido interações funcionais entre as espécies, oferecendo condições ecofisiológicas capazes de oferecer propriedades emergentes suficientes para abrigar novas espécies, na medida em que todas as demais condições do meio físico e geoambientais foram similares, bem como as condições de oferta de propágulos. A heterogeneidade ambiental dos ecossistemas aumenta a diversidade de espécies, influenciando na reprodução, no recrutamento, na especialização e na divisão dos recursos (Ruschel et al., 2009).

Os menores valores de riqueza na área testemunha (T0) estão relacionados provavelmente ao não plantio, embora haja também variação sazonal na colonização espontânea.

Os índices de diversidade de Shannon-Weaver $\left(\mathrm{H}^{\prime}\right)$ de espécies regenerantes entre os tratamentos e a área testemunha variaram de acordo com a as 
Tabela 3. Índice de diversidade de Shannon-Weaver ( $\left.\mathrm{H}^{\prime}\right)$, para os tratamentos $\mathrm{T} 1, \mathrm{~T} 2, \mathrm{~T} 3, \mathrm{~T} 4$, $\mathrm{T} 5$ e a área testemunha (T0) no período de inverno (2007) e verão (2008), em área de empréstimo na Ilha da Madeira, Itaguaí, RJ, reflorestada em 1994.

Table 3. Shannon-Weaver (H') diversity index, for treatments T1, T2, T3, T4, T5 and Testimony area (T0) on winter (2007) and summer (2008) period, at a reclamation area, Ilha da Madeira, Itaguaí, RJ, reforested on 1994.

\begin{tabular}{|ccc|} 
Tratamentos & Inverno & \multicolumn{2}{c}{ Verão } \\
\cline { 2 - 3 } \cline { 3 - 3 } T1 & $\mathbf{H}^{\prime}$ & H' $^{\prime}$ \\
T2 & 2,36 & 2,60 \\
T3 & 2,25 & 2,19 \\
T4 & 1,68 & 1,70 \\
T5 & 2,35 & 2,94 \\
\hline T0 (Testemunha) & 2,45 & 2,55 \\
\hline
\end{tabular}

estações (inverno e verão) (Tabela 3), embora sem se mostrarem estatisticamente diferentes. Esse fato pode ser devido ao tamanho reduzido do esforço amostral, condicionado pela pequena área com idênticas características geoambientais, mas os resultados sinalizam uma tendência de desenvolvimento dos ecossistemas, evidenciando que a capacidade de acúmulo de umidade do ecossistema é um fator limitante (Bazzaz \& Picket, 1980).

No período (inverno), o T1 $(2,36)$ apresentou o maior valor e T3 $(1,68)$, o menor. Esse resultado provavelmente se justifica também em função do plantio inicial do tratamento T1 (100\% de uma única espécie - A. auriculiformis); essas árvores, possivelmente após os dez primeiros anos em competição intraespecífica, foram extintas, transformando o ecossistema sem dossel e favorecendo a entrada de espécies anuais oportunistas (pioneiras) (Anexo 1), pois, segundo Larcher (2004), plantas anuais do tipo investidor usam seus assimilados para ganhar rapidamente uma abundante matéria orgânica, aproveitando um curto período favorável do ano para crescer, florescer e frutificar.

No período de verão, o T4 apresentou maior diversidade $(2,94)$ provavelmente em função do conjunto inicial de espécies implantadas no reflorestamento, composto por espécies de rápido crescimento (Lorenzi, 2002), que lhe conferiram, nos resultados silviculturais, maior DAP e maior altura individual. Além disso, possui espécies de dispersão zoocórica e uma espécie perenifólia (I. laurina), e duas semidecíduas (P. gonoacantha Mart. J. F. Macbr. e a $P$ guajava L), que podem ter atraído dispersores.
O tratamento T3 (1,70) apresentou valores menores em ambas as estações, provavelmente em função de o conjunto inicial ter sido predominantemente de espécies decíduas, deixando o substrato exposto e facilitando menos o estabelecimento de espécies colonizadoras.

Em comparação com trabalhos similares, os valores do índice de diversidade ( $\left.\mathrm{H}^{\prime}\right)$ encontrados neste estudo foram considerados altos. Segundo Neves (2004), na mesma área de estudo, após seis anos de implantação dos tratamentos, o maior valor encontrado foi de 1,23 no inverno para o tratamento T2 e 1,44 no verão para o T5. Os índices de diversidade também foram considerados altos, quando comparados com os encontrados por Cortines (2005) (H’ entre 1,25 a 2,11) e Roppa (2009) (H’ entre 0,68 a 1,99), em sítios edáficos similares e com densidades semelhantes, efetuados em 1995 no PRAD da Pedreira Vigné, no município de Nova Iguaçu, RJ. Isso indica uma tendência à sustentabilidade ambiental nas áreas em que os tratamentos foram implantados.

\section{CONCLUSÕES}

Após 14 anos da reabilitação, ocorreram diferenças na composição florística e na riqueza de espécies no estrato regenerante sob os reflorestamentos implantados como medidas biológicas de reabilitação de áreas degradadas, demonstrando evolução desses ecossistemas na busca da sustentabilidade ambiental, principalmente quando comparados com a área testemunha. 
O Tratamento T5 ( $\mathrm{n}=8$ espécies implantadas), com predomínio da Mimosa caesalpiniaefolia Benth (21\%), apresentou mais espécies no estrato regenerante na condição de oferta de umidade ampla (verão) e reduzida (inverno), apresentando-se com melhor desempenho. Enquanto que, no verão, o T3 ( $\mathrm{n}=4)$, predomínio de Acacia mangium Willd (30\%) e T4 $(n=6)$, predomínio de Acacia auriculiformis Sandw (22\%) no inverno, apresentaram os menores valores entre os tratamentos, sendo considerados como os menos indicados. Esses foram estatisticamente diferentes da T0 (testemunha), que apresentou os menores valores encontrados nesta pesquisa.

Houve evolução de todos os ecossistemas reabilitados na busca da sustentabilidade ambiental, quando comparados com a área testemunha (T0).

\section{AGRADECIMENTOS}

Ao Programa de Pós-Graduação em Ciências Ambientais e Florestais da Universidade Federal Rural do Rio de Janeiro (UFRRJ), ao Laboratório de Manejo de Bacias Hidrográficas (UFRRJ) e à Universidade do Estado da Bahia (UNEB).

\section{STATUS DA SUBMISSÃO}

Recebido: $21 / 12 / 2010$

Aceito: $17 / 10 / 2011$

Resumo publicado online: 26/10/2011

Artigo completo publicado: 22/12/2011

\section{AUTOR(ES) PARA CORRESPONDÊNCIA}

\section{Joana Farias dos Santos}

Programa de Ciências Ambientais e Florestais, Universidade Federal Rural do Rio de Janeiro - UFRRJ, BR 465, Km 7, CEP 23890-000, Seropédica, RJ, Brasil e-mail: joanafarias@yahoo.com.br

\section{REFERENNCIAS}

Angiosperm Phylogeny Group - APG II. An update of the Angiosperm Phylogeny Group classification for the orders and families of flowering plants: APG II. Botanical Journal of the Linneam Society 2003; 141:399-
436. http://dx.doi.org/10.1046/j.1095-8339.2003.t01-100158.x

Bazzaz FA, Pickett STA. Physiological ecology of tropical succession: a comparative review. Annual Review of Ecology Systematic 1980; 11:287-310. http:// dx.doi.org/10.1146/annurev.es.11.110180.001443

Bechara FC, Campos Filho EM, Barretto KD, Gabriel VA, Antunes AZ, Reis A. Unidades Demonstrativas de Restauração Ecológica através de Técnicas Nucleadoras de Biodiversidade. Revista Brasileira de Biociências 2007; 5(supl. 1):9-11.

Brower JE, Zar JH. Field \& laboratory methods for general ecology. 2rd ed. Iowa: Wm. C. Brown Publishers, Dubuque; 1984.226 p.

Cortines E. Avaliação da sustentabilidade ecológica de plantios realizados em ecossistemas perturbados, Nova Iguaçu-RJ [monografia]. Seropédica: Instituto de Biologia, Universidade Federal do Rio de Janeiro, 2005.

Jones CG, Lawton JH, Shachak M. Positive and negative effects of organisms as physical ecosystem engineers. Ecology 1997; 78(7):1946-1957. http://dx.doi. org/10.1890/0012-9658(1997)078[1946:PANEOO]2.0. $\mathrm{CO} ; 2$

Larcher W. Ecofisiologia vegetal. São Carlos: RiMa; 2004. $531 \mathrm{p}$.

Lorenzi, H. Árvores Brasileiras: manual de identificação e cultivo de plantas arbóreas nativas do Brasil. 2rd ed. Nova Odessa: Plantarum, 2002. vol. 2.

Macedo AC. Revegetação: matas ciliares e de proteção ambiental. São Paulo: Fundação Florestal; 1993. 27 p.

Mantovani W. Análise florística e fitossociológica do estrato herbáceo-subarbustivo do cerrado na reserva biológica de Mogi Guaçu e em Itirapina, SP [tese]. Campinas: Universidade Estadual de Campinas; 1987. $166 \mathrm{p}$.

Mattos CCLV, Silva MAR, Oliveira MN, Combat IB. Boletim agrometeorológico, UFRRJ-1996. Floresta $e$ Ambiente 1998; 5(1):208-215.

Moraes LFD, Assumpção JM, Luchiare C, Pereira TS. Plantio de espécies arbóreas nativas para a restauração ecológica na Reserva Biológica de Poço das Antas. Rodriguésia 2006; 57(3):477-489.

Neves LG. Eficiência conservacionista de medidas biológicas em reabilitação de áreas degradadas no domínio ecológico da Mata Atlântica [dissertação]. Seropédica: Universidade Federal Rural do Rio de Janeiro; 2004. 126 p.

Pinheiro CAA. Dinamismo dos processos erosivos em fontes pontuais de emissão de sedimentos para a baía de Sepetiba [dissertação]. Seropédica: Universidade Federal Rural do Rio de Janeiro; 2004. 67 p. 
R Development Core Team. R Foundation for Statistical Computing. Vienna; 2007. [cited 2010 maio 3]. AvaAvailable from: http://www.r-project.org.

Rio de Janeiro (Estado). Zoneamento Econômico Ecológico - ZEE. Projeto 1: Diagnóstico Ambiental da Bacia Hidrográfica da Baia de Sepetiba. Rio de Janeiro: Secretaria Estadual do Meio Ambiente do Estado do Rio de Janeiro; 1996. CD-ROM.

Ruschel AR, Mantovani M, Reis MS, Nodari RO. Caracterização e dinâmica de duas fases sucessionais em floresta secundária da Mata Atlântica. Revista Árvore 2009; 33(1):101-115. http://dx.doi.org/10.1590/ S0100-67622009000100011

Roppa C. Avaliação da dinâmica de restauração de ecossistemas perturbados da Mata Atlântica em uma região de exíguos atributos ambientais, Nova Iguaçu - RJ [dissertação]. Seropédica: Universidade Federal Rural do Rio de Janeiro; 2009.
Universidade Federal Rural do Rio de Janeiro - UFRRJ. Plano de Recuperação de Áreas de Empréstimo da Serviços de Engenharia Rodoférrea S. A. Itaguaí: UFRRJ; 1993. 79 p. Relatório Final.

Valcarcel R, D’altério CF. Medidas físico-biológicas de recuperação de áreas degradadas: avaliação das modificações edáficas e fitossociológicas. Floresta $e$ Ambiente 1998; 5(1):68-88.

Valcarcel R, Silva Z. A eficiência conservacionista de medidas de recuperação de áreas degradadas: proposta metodológica. Floresta 2000; 27(1):101-114.

Valcarcel R, Valente FDW, Morokawa MJ, Cunha Neto FV, Pereira CR. Avaliação da biomassa de raízes finas em área de empréstimo submetida a diferentes composições de espécies. Revista Árvore 2007; 31(5):923-930. http:// dx.doi.org/10.1590/S0100-67622007000500016

Zar JH. Biostatistical analysis. 4rd ed. New Jersey: Prentice-Hall, Inc.; 1999. 663 p. 
Anexo 1. Relação de espécies amostradas nos tratamentos T1, T2, T3, T4, T5 e na testemunha T0, em área de empréstimo na Ilha da Madeira, RJ, reflorestada em 1994, em ordem alfabética de família, gênero e espécie, com os respectivos nomes populares e hábitos: “ઋ” corresponde ao período de inverno (estação seca) e "x" ao período de verão (estação chuvosa).

Atach 1. Relation of surveyed species on treatments T1,T2,T3,T4, T5 and withness T0, on a reclamation area at Ilha da Madeira, RJ, reforested on 1994, on alphabetic order of families, genus and species, with respective popular names, habit: “*” corresponds to dry period (winter) and " $x$ " to rainy period (summer).

\begin{tabular}{|c|c|c|c|c|c|c|}
\hline Família/Nome Científico & Nome Popular & T0 T1 & T2 & T3 & T4 & T5 \\
\hline \multicolumn{7}{|l|}{ ANACARDIACEAE } \\
\hline Astronium graveolens Jacq. & guaritá & & & & $\mathrm{x}$ & $\mathrm{x}$ \\
\hline Morfoespécie 05 & - & & & & & * \\
\hline Morfoespécie 11 & - & & & & & $\mathrm{x}$ \\
\hline \multicolumn{7}{|l|}{ ANEMIACEAE } \\
\hline Anemia phyllitidis (L.) Sw. & - & & & & $\mathrm{x}$ & $\mathrm{x}$ \\
\hline Anemia villosa Humb.Obonpl. ex Wild. & - & & & & $\mathrm{x}$ & \\
\hline \multicolumn{7}{|l|}{ ANNONACEAE } \\
\hline Rollinia laurifolia Schltdl. & - & & & $\mathrm{x}$ & & \\
\hline \multicolumn{7}{|l|}{ APOCYNACEAE } \\
\hline Ditassa sp. & - & $\mathrm{x}$ & & * & & \\
\hline Himatanthus bracteatus (A. DC.) Woodson & - & & & & $\mathrm{x}$ & \\
\hline Oxypetalum sp. & & $\mathrm{x}$ & & & & \\
\hline Oxypetalum cordifolium (Vent.) Schltr & - & & ${ }^{\star} \mathrm{x}$ & & & \\
\hline
\end{tabular}

\section{ARISTOLOCHIACEAE}

Aristolochia ruiziana (Kl.) Duch.

\section{ASTERACEAE}

Adenostemma brasilianum (Pers.) Cass.

Baccharis sp.

Baccharis punctigera DC.

Baccharis dracunculifolia (Less) DC.

Baccharis serrutata (Lam.) Person

Baccharis trinervus (Lam.) Pers.

papo-de-peru

Clibadium rotundifolium DC.

Elephontopus sp.

Elephantopus angustifolius Sw.

Elephantopus mollis Kunth

Eupatorium laevigatum Lam.

Gnaphalium purpureum L.

Mikania glomerata Spreng.

Mikania Laevis DC.

Mikania congesta

Piptocarpha quadrangularis (Vell.) Baker

Vernonia macrophylla Less.

Vernonia polyanthes Less.

Vernonia scorpioides (Lam.) Pers.

Vernonia squamosa Gardn.

\section{BIGNONIACEAE}

Anrabiodaea leucopogon

Cybistax antisyphilitica (Mart.) Mart.

Macfadyena unguis-cati (L.) A. Gentry

Tabebuia umbellata (Sond.) Sandwith

Morfoespecie 08

cravinho do mato

tecrim- $x$

alecrim-do-campo $\quad{ }^{*} \mathrm{x}{ }^{*} \mathrm{x}$

assa-peixe-branco

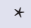

${ }^{*} \mathrm{X} \mathrm{X}$

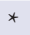

*

\section{$\mathrm{X}$}

língua -de-vaca mata-pasto

Marcela folha fina

Guaco

Morfoespecie 09

ipê-verde

unha-de-gato

ipê-amarelo

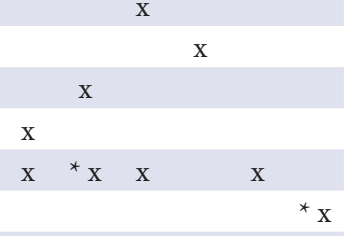

$\mathrm{X}$

${ }^{*} \mathrm{X} \quad \mathrm{X} \quad{ }^{*} \mathrm{X}$

${ }^{*} \mathrm{x} \quad{ }^{*} \mathrm{x} \quad{ }^{*} \mathrm{x} \quad \mathrm{x} \quad{ }^{*} \mathrm{x}$

${ }^{*} \mathrm{X}$

${ }^{*} \mathrm{x} \quad{ }^{*} \mathrm{x} \quad{ }^{*} \mathrm{x} \quad \mathrm{x}$

$\begin{array}{lll}\mathrm{X} & \mathrm{X} & \mathrm{X}\end{array}$

*

${ }^{*} \mathrm{x} \quad * \mathrm{x} \quad \mathrm{x}$

* * $\quad$ * $\mathrm{x}$

$\mathrm{x}$ 
Anexo 1. Continuação...

Atach 1. Continued...

\begin{tabular}{|c|c|c|c|c|c|c|c|}
\hline Família/Nome Científico & Nome Popular & T0 & T1 & T2 & T3 & T4 & T5 \\
\hline \multicolumn{8}{|l|}{ BLECHUACEAE } \\
\hline Blechnum occidentale $\mathrm{L}$. & - & & & & & $\mathrm{x}$ & \\
\hline \multicolumn{8}{|l|}{ BORAGINACEAE } \\
\hline Cordia hypoleuca A. DC. & - & & $\mathrm{x}$ & ${ }^{*} \mathrm{x}$ & $\mathrm{x}$ & & $\mathrm{x}$ \\
\hline Cordia leucocephala Moric. & muleque-duro & & & * & & & \\
\hline Cordia trichoclada DC. & louro-tabaco & $\mathrm{x}$ & & & & & \\
\hline \multicolumn{8}{|l|}{ CANNABACEAE } \\
\hline Trema micrantha (L.) Blume & crindiúva; grandiuva & & & ${ }^{*} \mathrm{x}$ & & & \\
\hline \multicolumn{8}{|l|}{ CYPERACEAE } \\
\hline Rhynchospora exaltata Kunth & capim-navalha & ${ }^{*} \mathrm{x}$ & $\mathrm{x}$ & & & & \\
\hline \multicolumn{8}{|l|}{ ERYTHROXILACEAE } \\
\hline Erythroxilum pulchrum A. St. -Hil. & arco-de-pipa & & & $\mathrm{x}$ & & & ${ }^{*} \mathrm{x}$ \\
\hline \multicolumn{8}{|l|}{ EUPHORBIACEAE } \\
\hline Croton floribundus Spoeng. & capixingui & & & & & $\mathrm{x}$ & \\
\hline Dalechampia sp. & - & & ${ }^{\star} \mathrm{x}$ & & & $\mathrm{x}$ & \\
\hline Dalechampia scandens $\mathrm{L}$. & cipó-fogo & & & & ${ }^{*} \mathrm{x}$ & & $\mathrm{x}$ \\
\hline Morfoespecie 07 & - & & & $\mathrm{x}$ & & & \\
\hline \multicolumn{8}{|l|}{ FABACEAE-CAESALPINIOIDEAE } \\
\hline Caesalpinia sp. & - & & & $\mathrm{x}$ & & * & \\
\hline \multicolumn{8}{|l|}{ FABACEAE-FABOIDEAE } \\
\hline Machaerium aculeatum Raddi & bico-de-pato & & & $\mathrm{x}$ & & * & \\
\hline Machaerium hirtum (Vell.) Stellfeld & Jacarandá-de-espinhos, bico-de-andorinha & & & * & & & \\
\hline Machaerium nyctitans (Vell.) Benth. & Jacarandá- ferro & & & & & $\mathrm{x}$ & \\
\hline Morfoespecie 10 & - & & & & & $\mathrm{x}$ & \\
\hline \multicolumn{8}{|l|}{ FABACEAE-MIMOSOIDEAE } \\
\hline Acacia polyphylla DC. & manjoleiro, maricá & $\mathrm{x}$ & $\mathrm{x}$ & $x^{*}$ & & $\mathrm{x}$ & \\
\hline Albizia lebbeck (L) Benth & albízia & & $\mathrm{x}$ & & $\mathrm{x}$ & & \\
\hline Albizia polycephala (Benth.) Killip Record & manjolo & $\mathrm{x}$ & & & & $\mathrm{x}$ & \\
\hline Inga laurina (Sw.) Willd & ingá & & & & & ${ }^{\star} \mathrm{x}$ & \\
\hline Leucaena leucocephala (Lam.) & leucena & & ${ }^{*} \mathrm{x}$ & ${ }^{*} \mathrm{x}$ & ${ }^{*} \mathrm{x}$ & $\mathrm{x}$ & \\
\hline Mimosa caesalpiniaefolia Benth. & sabiá & & & ${ }^{*} \mathrm{x}$ & ${ }^{\star} \mathrm{x}$ & ${ }^{\star} \mathrm{x}$ & \\
\hline Piptadenia gonoacantha (Mart.) J. F. Macbr. & pau-jacaré & & & ${ }^{*} \mathrm{x}$ & & $\mathrm{x}$ & ${ }^{*} \mathrm{x}$ \\
\hline Morfoespécie 04 & - & & & * & & & * \\
\hline Morfoespécie 06 & - & & $\mathrm{x}$ & & & & $\mathrm{x}$ \\
\hline Morfoespécie 12 & - & & & & & & $\mathrm{x}$ \\
\hline \multicolumn{8}{|l|}{ FABACEAE-PAPILIONOIDEAE } \\
\hline Canavalia ensiformis (L.) DC. & feijão-de-porco & & $\mathrm{x}$ & ${ }^{*} \mathrm{x}$ & ${ }^{*} \mathrm{x}$ & ${ }^{\star} \mathrm{x}$ & \\
\hline Cajanus cajan (L.) Millsp. & feijão guandu; feijão-andu & & $\mathrm{x}$ & & & & \\
\hline Myrocarpus frondosus Allemão & cabreúva & & & & * & & \\
\hline Vigna lasiocarpa (Mart.ex Benth.) Verdc. & - & & $\mathrm{x}$ & & & & \\
\hline \multicolumn{8}{|l|}{ GLEICHNIACEAE } \\
\hline Gleichenella pectinata (Willd.) Ching & samambaia-de-barranco & ${ }^{*} \mathrm{x}$ & & & & & \\
\hline \multicolumn{8}{|l|}{ LAMIACEAE } \\
\hline Hyptis lophanta Mart. ex Benth. & hortelã & & & & & * & \\
\hline Hyptis mutabilis (Rich.) Briq. & - & & & ${ }^{*} \mathrm{x}$ & & & \\
\hline \multicolumn{8}{|l|}{ LAURACEAE } \\
\hline Endlicheria paniculata (Spreng) Macber. & - & & $\mathrm{x}$ & ${ }^{*} \mathrm{x}$ & & & \\
\hline Nectandra membranacea (Swartz) Griseb. & canela-branca & & & & ${ }^{\star} \mathrm{x}$ & $\mathrm{x}$ & ${ }^{\star} \mathrm{x}$ \\
\hline
\end{tabular}


Anexo 1. Continuação...

Atach 1. Continued...

Família/Nome Científico

Nome Popular

$\begin{array}{llllll}\text { T0 } & \text { T1 } & \text { T2 } & \text { T3 } & \text { T4 } & \text { T5 }\end{array}$

\section{LYGUDIACEAE}

Lygodium volubile Sw.

abre-caminho

MALPIGHIACEAE

Banisteriopsis sp.

Stigmaphyllon af. Ciliatum A. Juss.

Morfoespecie 13

MALVACEAE

Luehea divaricata Mart.

Waltheria americana L.

Waltheria indica $\mathrm{L}$.

Waltheria sp.

Wissadula sp.

\section{MARANTACEAE}

Ctenanthe cf. pilosa

MELASTOMATACEAE

Clidemia hirta (L.) D. Don

Clidermia sylvestris Sw.

Tibouchina granulosa (Desr.) Cogn.

Morfoespécie 01

Morfoespécie 03

\section{MELIACEAE}

Guarea guidonia (L.) Sleumer

Guarea macrophylla Vahl.

Trichilia lepidofa Mart.

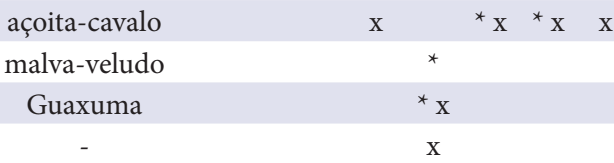

$\mathrm{X}$

${ }^{*} \mathrm{x} \quad{ }^{*} \mathrm{x}$

Morfoespécie 02

Morfoespécie 14

MONIMIACEAE

Mollinedia sp.

MYRTACEAE

Campomanesia sp.

Eugenia sp.

Myrcia splendens (Sw.) DC.

PASSIFLORACEAE

Passiflora biflora

PIPERACEAE

Piper sp.

Piper aduncum L.

Piper anisum (Spreng.) Angely

Piper arboreum Aubl.

Piper mollicomum Kunth

Piper solmsianum C. DC.

\section{POACEAE}

Andropogon bigornis L.

Andropogon selloanus (Hackel)Hackel

Bambusa vulgaris Schrad.

Brachiaria plantaginea (Link)Hitch

Cynodon plectostacyus (K. Schum) Pilg.

Imperata brasiliensis Trin.

$\begin{array}{ccc}\begin{array}{c}\text { carrapeta } \\ \text { catiguá-morcego }\end{array} & { }^{*} \mathrm{x}{ }^{*} \mathrm{x}{ }^{*} \mathrm{x} \quad{ }^{*} \mathrm{x}{ }^{*} \mathrm{x} \\ \text { catiguá } & \mathrm{x} & { }^{*} \mathrm{x} \\ - & & { }^{*} \\ - & & \mathrm{x}\end{array}$

quaresmeira $\quad{ }^{*} \mathrm{x}$

*

$\mathrm{x}$

$\mathrm{x}$

${ }^{*} \mathrm{X}$

\begin{tabular}{|c|c|c|c|c|c|}
\hline & \multicolumn{5}{|c|}{$\mathrm{x}$} \\
\hline pimenta-de-macaco & ${ }^{*} \mathrm{X}$ & ${ }^{*} \mathrm{x}$ & ${ }^{*} \mathrm{x}$ & * & ${ }^{*} \mathrm{x}$ \\
\hline- & & & & & $\mathrm{x}$ \\
\hline Pimenta-de-macaco & & & & $\mathrm{x}$ & $\mathrm{x}$ \\
\hline pariparoba, jaguarandi & & & & $\mathrm{x}$ & $\mathrm{x}$ \\
\hline jaborandi & & $*$ & & & * \\
\hline
\end{tabular}

capim-rabo-de-burro

capim-barba-de-bode

bambu

capim-papuã

grama estrela

capim-sapé

${ }^{*} \mathrm{x}$ 
Anexo 1. Continuação...

Atach 1. Continued...

\section{Família/Nome Científico}

Melinis minutiflora P. Beauv

Olyra micrantha H.B.K.

Panicum maximum Jacq.

Panicum pilosum Sw.

Paspalum sp.

Paspalum notatum Flüggé

Pharus lappulaceus Aubl.

\section{POLIGALACEAE}

Poygala sp.

\section{RUBIACEAE}

Psychotria sp.

Psychotria leiocarpa Cham. \& Schltdl.

Spermacoce verticillata $\mathrm{L}$.

\section{SALICACEAE}

Casearia decandra Jacq.

Casearia sylvestris Sw.

\section{SAPINDACEAE}

Cupania oblongifolia Mart.

Paullinia turbacensis Kunth

Serjania caracasana (Jacq.) Willd.

Serjania cuspidata Cambess.

Serjania communis Cambess.

\section{SIPARUNACEAE}

Siparuna guianensis Aubl.

\section{SMILACACEAE}

Smilax fluminensis Stend.

Smilax quinquenervia Vell.

\section{SOLANACEAE}

Solanum americanum Mill.

Solanum argenteum Duanl

Solanum asperum L. C. Rich.

Solanum granulosoleprosum Dunal

Solanum paniculatum L.

Solanum pseudoquina A. St. -Hil.

\section{TRIGONIACEAE}

Trigonia villosa Aubl.

\section{URTICACEAE}

Cecropia pachystachya Trec.

\section{VERBENACEAE}

Gmelina arborea Roxb.

Lantana sp.

Lantana camara $\mathrm{L}$.

Lantana canescens Kunth

VITACEAE

Cissus sp.

Cissus verticillata (L.) Nicolson

\section{Nome Popular}

capim-gordura

taquara

capim-colonião

grama-batatais

\section{$\mathrm{X}$}

$\mathrm{X}$

$\mathrm{x}$

* $\mathrm{X}$

café-do-mato; grandiúva-de-anta

vassourinha-de-botão

$\mathrm{X}$

$\mathrm{X}$

$$
\text { guaçatunga }
$$

espeteiro; pau de lagarto

${ }^{*} \mathrm{X}$

$\mathrm{x}$

\begin{tabular}{|c|c|c|c|c|}
\hline camboatá & $\mathrm{x}$ & $\mathrm{x}$ & $\mathrm{x}$ & ${ }^{*} \mathrm{X}$ \\
\hline- & & $\mathrm{x}$ & & \\
\hline - & ${ }^{*} \mathrm{X}$ & * & ${ }^{*} \mathrm{X}$ & ${ }^{*} \mathrm{X}$ \\
\hline - & $\mathrm{X}$ & ${ }^{*} \mathrm{X}$ & & \\
\hline - & $\mathrm{x}$ & $\mathrm{X}$ & & \\
\hline
\end{tabular}

erva-santa

salsaparrilha

japecanga

${ }^{*} \mathrm{X} \quad{ }^{*} \mathrm{X}$

maria-pretinha

Solanum- prata

coça-coça

fumo-bravo

jurubeba

coerana

$\mathrm{X}$

${ }^{*} \mathrm{X}$

embaúba

${ }^{*} \mathrm{x}$

cambará; camará-de-espinho

$\mathrm{X}$

cambarazinho; camara

$\mathrm{X}$ 